\title{
Blow-up theory for symmetric critical equations involving the $p$-Laplacian
}

\author{
Nicolas SAINTIER \\ Departamento de Matemática, FCEyN, \\ Universidad de Buenos-Aires, \\ Pabellón I, Ciudad Universitaria \\ (1428), Buenos Aires, Argentina. \\ e-mail: saintier@math.jussieu.fr, nsaintie@dm.uba.ar
}

\begin{abstract}
We describe in this paper the asymptotic behaviour in Sobolev spaces of sequences of solutions of critical equations involving the $p$-Laplacian (see equations $\left(E_{\alpha}\right)$ below) on a compact Riemannian manifold $(M, g)$ which are invariant by a subgroup of the group of isometries of $(M, g)$. We also prove pointwise estimates.
\end{abstract}

2000 Mathematics Subject Classification: 35B40, 35J20, 35B33

Key words: $p$-Laplacian, blow-up, invariance under isometries

Let $(M, g)$ be a smooth compact Riemannian $n$-manifold, $G$ a closed subgroup of the group of isometries $\operatorname{Isom}_{g}(M)$ of $(M, g)$ and $k=\min _{x \in M} \operatorname{dim} G x$, where $G x$ denotes the orbit of a point $x \in M$ under $G$. We say that a function $\psi: M \rightarrow \mathbb{R}$ is $G$-invariant if $\psi(g x)=\psi(x)$ for any $x \in M$ and $g \in G$. We consider equations like

$$
\Delta_{p, g} u+h_{\alpha} u^{p-1}=f u^{p^{*}-1}
$$

where $1<p<n-k, \Delta_{p, g} u=-\operatorname{div}_{g}\left(|\nabla u|_{g}^{p-2} \nabla u\right)$ is the $p$-Laplacian of $u$, $p^{*}=\frac{(n-k) p}{n-k-p}$ is the critical exponent for the injection from the Sobolev space $H_{1, G}^{p}(M)$ of $G$-invariant functions in $L^{p}(M)$ whose gradient is also in $L^{p}(M)$, into the Lebesgue spaces $L_{G}^{q}(M)$ of $G$-invariant functions in $L^{q}(M)$ (cf HebeyVaugon [6]), $f$ is a $C^{1} G$-invariant function, and $\left(h_{\alpha}\right)$ is a sequence of continuous $G$-invariant functions converging uniformly to some continous $G$-invariant function $h_{\infty}$. The solutions we consider are in $H_{1}^{p}$; therefore, a solution to $\left(E_{\alpha}\right)$ has to be taken in the distribution sense. We assume that the operator $\Delta_{p, g}+h_{\infty}$ is coercive 
in the sense that there exists $\lambda>0$ such that for all $u \in H_{1, G}^{p}(M)$,

$$
\int_{M}\left(|\nabla u|_{g}^{p}+h_{\infty}|u|^{p}\right) d v_{g} \geq \lambda\|u\|_{H_{1}^{p}}^{p}
$$

In fact, we can easily prove that $\Delta_{p, g}+h_{\infty}$ is coercive if and only if there exists $\lambda>0$ such that for all $u \in H_{1, G}^{p}(M)$,

$$
\int_{M}\left(|\nabla u|_{g}^{p}+h_{\infty}|u|^{p}\right) d v_{g} \geq \lambda\|u\|_{p}^{p}
$$

A necessary condition for $\left(E_{\alpha}\right)$ to admit a positive solution $u$ is $\max _{M} f>0$. Indeed, multiplying $\left(E_{\alpha}\right)$ by $u$, integrating by parts and using the coercivity assumption (1) yields

$$
\int_{M} f u^{p^{*}} d v_{g} \geq \lambda\left\|u_{\alpha}\right\|_{H_{1}^{p}}^{p}+o(1) .
$$

We then deduce that $f$ must be positive somewhere, and then $\max _{M} f>0$. From now on, we assume that $\max _{M} f>0$. We also consider the limit equation obtained by letting formally $\alpha \rightarrow+\infty$ in $\left(E_{\alpha}\right)$, namely

$$
\Delta_{p, g} u+h_{\infty} u^{p-1}=f u^{p^{*}-1} .
$$

For each $\alpha$, let $u_{\alpha}$ be a $G$-invariant weak positive solution of $\left(E_{\alpha}\right)$ and assume that the sequence $\left(u_{\alpha}\right)$ is bounded in $H_{1}^{p}$. The purpose of this note is to describe the asymptotic behavior of the $u_{\alpha}$ 's. In the case where the group $G$ is reduced to the identity, it is known (see Saintier [9], Hebey-Robert [5], Struwe [10]) that $u_{\alpha}$ can be written as the sum of a weak solution of the limit equation $\left(E_{\infty}\right)$ plus a finite sum of "bubbles" plus a sequence of functions converging strongly to 0 in $H_{1}^{p}$. A bubble is a sequence of functions obtained by rescaling positive solution of the Euclidean critical equation $\Delta_{p, \xi} u=u^{q-1}$ in $\mathbb{R}^{n}, q=n p /(n-p)$, where $\xi$ is the Euclidean metric on $\mathbb{R}^{n}$. We prove here (cf the theorem below) that this decomposition still holds in the context of $G$-invariant functions under some assumptions on the orbits of $G$ (assumption $(\mathrm{H})$ below) and with an extended notion of bubble.

We now recall some known facts and fix some notations. We refer to Bredon [1] for more details (see also Hebey-Vaugon [6] and Faget [2]). Let $G^{\prime}$ be a closed subgroup of $\operatorname{Isom}_{g}(M)$. Then $G^{\prime}$ is a Lie group. For each $x \in M$, we let $\bar{x}=\Pi(x)$, where $\Pi: M \rightarrow M / G^{\prime}$ is the canonical surjection, and denote by $G^{\prime} x=\{g x, g \in$ $\left.G^{\prime}\right\}$ (resp. $S_{x}=\left\{g \in G^{\prime}, g x=x\right\}$ ) the orbit (resp. the stabilizator) of $x$ under the action of $G^{\prime}$. Then $G^{\prime} x$ is a compact submanifold of $M$ naturally isomorphic to the quotient group $G^{\prime} / S_{x}$. An orbit $G^{\prime} x$ is said principal if its stabilizator is minimal up to conjugacy i.e. for all $y \in M, S_{y}$ contains a subgroup conjugate to $S_{x}$. In particular, the principal orbits are of maximal dimension (but the converse is false). If we denote by $\Omega$ the union of all the principal orbits, then $\Omega$ is a dense 
open subset of $M$ and $\Omega / G^{\prime}$ is a smooth connected manifold which can be equiped with a Riemaniann metric $\bar{g}$ in such a way that the canonical surjection from $\Omega$ to $\Omega / G^{\prime}$ is a Riemannian submersion. We then consider the metric $\tilde{g}$ belonging to the conformal class of $\bar{g}$ defined by

$$
\tilde{g}=\bar{v}^{\frac{2}{n-k-p}} \bar{g}
$$

where $\bar{v}(\bar{x})=\operatorname{Vol}\left(\Pi^{-1}(\bar{x})\right)=\operatorname{Vol}\left(G^{\prime} x\right)$ denotes the volume of $G^{\prime} x$ computed with respect to the induced metric. We will denote by $B_{\bar{x}}^{\bar{g}}(r)$ and $B_{\bar{x}}^{\tilde{g}}(r)$ the geodesic balls centered at $\bar{x}$ of radius $r$ for the metric $\bar{g}$ and $\tilde{g}$ respectively. Given a Riemannian manifold $N$, we denote by $H_{1}^{p}(N)$ the usual Sobolev space of functions $u \in L^{p}(N)$ such that $\nabla u \in L^{p}(N)$ with the norm $\|u\|_{H_{1}^{p}}^{p}=\|u\|_{p}^{p}+\|\nabla u\|_{p}^{p}$, and by $\stackrel{\circ}{H_{1}^{p}}(N)$ the closure of $C_{c}^{\infty}(N)$ for the norm $\|\cdot\|_{H_{1}^{p}}$. If $G^{\prime}$ is a subgroup of isometries of $N$, we let $L_{G^{\prime}}^{p}(N), H_{1, G^{\prime}}^{p}(N)$ and $\stackrel{\circ}{H_{1, G^{\prime}}^{p}}(N)$ be the space of $G^{\prime}$-invariant functions in $L^{p}(N), H_{1}^{p}(N)$ and $\stackrel{\circ}{H_{1}^{p}}(N)$ respectively:

$$
\begin{aligned}
& L_{G^{\prime}}^{p}(N)=\left\{u \in L^{p}(N) \text { s.t. } \forall g \in G^{\prime}, u(g x)=u(x) \text { a.e. in } N\right\}, \\
& H_{1, G^{\prime}}^{p}(N)=\left\{u \in H_{1}^{p}(N) \text { s.t. } \forall g \in G^{\prime}, u(g x)=u(x) \text { a.e. in } N\right\} \text {, } \\
& \stackrel{\circ}{H_{1, G^{\prime}}^{p}}(N)=\left\{u \in \stackrel{\circ}{H_{1}^{p}}(N) \text { s.t. } \forall g \in G^{\prime}, u(g x)=u(x) \text { a.e. in } N\right\} \text {, }
\end{aligned}
$$

We assume that $k=\min _{x \in M} \operatorname{dim} G x \geq 1$ and make the following assumption on the $G$-orbits of dimension $k$ :

(H) for each $G$ - orbit $G x_{0}$ of minimal dimension $k$, there exist $\delta>0$ and a closed subgroup $G^{\prime}$ of $\operatorname{Isom}_{g}(M)$ such that

$$
G^{\prime} x_{0}=G x_{0}
$$

and, for all $x \in B_{G x_{0}}(\delta):=\left\{y \in M, d_{g}\left(y, G x_{0}\right)<\delta\right\}$,

$$
G^{\prime} x \text { is principal and } G^{\prime} x \subset G x .
$$

We refer to Faget [2] for examples of manifolds and groups satisfying $(\mathrm{H})$. In particular, $\operatorname{dim} G^{\prime} x=\operatorname{dim} G x_{0}=k$ for all $x \in B_{G x_{0}}(\delta)$ and we can consider the Riemannian quotient $(n-k)$-manifold $N:=B_{G x_{0}}(\delta) / G^{\prime}$. We fix a smooth cut-off function $\eta \in C_{c}^{\infty}\left(\mathbb{R}^{n-k}\right)$ with support in $B_{0}(2)$ such that $0 \leq \eta \leq 1$ and $\eta \equiv 1$ in $B_{0}(1)$. Given $\bar{x}_{1} \in N$ and $\delta^{\prime} \in\left(0, i_{\tilde{g}}\left(\bar{x}_{1}\right) / 2\right)$, we let

$$
\eta_{\bar{x}_{1}, \delta^{\prime}}(\bar{x})=\eta\left(\frac{d_{\tilde{g}}\left(\bar{x}_{1}, \bar{x}\right)}{\delta^{\prime}}\right)
$$

for $\bar{x} \in N$. Here, $i_{\tilde{g}}\left(\bar{x}_{1}\right)$ denotes the injectivity radius of $N$ at $\bar{x}_{1}$.

We now define a bubble in this context. Let $\left(x_{\alpha}\right)$ be a sequence of points in $M$ converging to some $x_{0} \in M$ such that $G x_{0}$ is of dimension $k$. Then assumption 
(H) provides us with a subgroup $G^{\prime}$ of $\operatorname{Isom}_{g}(M)$ and a $\delta>0$ such that (H1) and (H2) hold. Let $2 \delta^{\prime}>0$ be inferior to the injectivity radius of the quotient $(n-k)$-manifold $N:=B_{G x_{0}}(\delta) / G^{\prime}$. Consider also a sequence $\left(R_{\alpha}\right) \subset[0,+\infty)$ such that $R_{\alpha} \rightarrow+\infty$. Given a positive solution $u \in H_{1}^{p}\left(\mathbb{R}^{n-k}\right)$ of the Euclidean equation

$$
\Delta_{p, \xi} u=f\left(x_{0}\right) \operatorname{Vol}\left(G x_{0}\right)^{\frac{-p}{n-k-p}} u^{p^{*}-1},
$$

where $\xi$ is the Euclidean metric, we define a bubble $\left(\bar{B}_{\alpha}\right)$ of centers $\left(\bar{x}_{\alpha}\right)$ and weights $\left(R_{\alpha}\right)$ in the usual way by

$$
\bar{B}_{\alpha}(\bar{x})=\eta_{\bar{x}_{\alpha}, \delta^{\prime}}(\bar{x}) R_{\alpha}^{\frac{n-k-p}{p}} u\left(R_{\alpha} \exp _{\bar{x}_{\alpha}}^{-1}(\bar{x})\right), \bar{x} \in N .
$$

where $\exp$ is the exponential map of $N$ for the metric $\tilde{g}$. We then define a bubble $B=\left(B_{\alpha}\right)$ of centers $\left(x_{\alpha}\right)$ and weights $\left(R_{\alpha}\right)$ as the $G^{\prime}$-invariant function satisfying

$$
B_{\alpha}=\bar{B}_{\alpha} \circ \Pi
$$

where $\Pi: B_{G x_{0}}(\delta) \rightarrow N$ is the canonical surjection. A generalized bubble is defined in the same way by considering a nontrivial, not necessarily positive, solution $u \in H_{1}^{p}\left(\mathbb{R}^{n-k}\right)$ of the Euclidean equation

$$
\Delta_{p, \xi} u=f\left(x_{0}\right) \operatorname{Vol}\left(G x_{0}\right)^{\frac{-p}{n-k-p}}|u|^{p^{*}-2} u .
$$

This definition clearly extends the usual definition of a bubble to the case of $G$-invariant functions. We also define the energy $E(B)$ of the (generalized) bubble $B$ by

$$
E(B)=\frac{1}{p} \int_{\mathbb{R}^{n-k}}|\nabla u|_{\xi}^{p} d x-\frac{f\left(x_{0}\right) \operatorname{Vol}\left(G x_{0}\right)^{-\frac{p}{n-k-p}}}{p^{*}} \int_{\mathbb{R}^{n-k}}|u|^{p^{*}} d x .
$$

We can prove as in Saintier ([9] step 1.5) that

$$
E(B) \geq f\left(x_{0}\right)^{-\frac{n-k-p}{p}} \operatorname{Vol}\left(G x_{0}\right) \frac{1}{n-k} K(n-k, p)^{k-n}
$$

where $K(n-k, p)$ denotes the best Sobolev constant for the injection of $H_{1}^{p}\left(\mathbb{R}^{n-k}\right)$ into $L^{p^{*}}\left(\mathbb{R}^{n-k}\right)$, namely

$$
\frac{1}{K(n-k, p)}=\inf _{u \in C_{c}^{\infty}\left(\mathbb{R}^{n-k}\right) \backslash\{0\}} \frac{\int_{\mathbb{R}^{n-k}}|\nabla u|_{\xi}^{p} d x}{\left(\int_{\mathbb{R}^{n-k}}|u|^{p^{*}} d x\right)^{p / p^{*}}}>0 .
$$

If we denote by $A$ the minimum volume of $G$-orbit of dimension $k$, we then have the minoration

$$
E(B) \geq\left(\max _{M} f\right)^{-\frac{n-k-p}{p}} A \frac{1}{n-k} K(n-k, p)^{k-n}
$$

which holds for any generalized bubble. 
Our result is then the following:

Theorem Let $(M, g)$ be a Riemaniann manifold, $G$ a closed subgroup of $\operatorname{Isom}_{g}(M)$ satisfying $(H)$ and $\left(u_{\alpha}\right)$ be a sequence of positive $G$-invariant solutions of $\left(E_{\alpha}\right)$ bounded in $H_{1}^{p}(M)$. There exist $u^{0} \in H_{1, G}^{p}(M)$ such that either $u^{0} \equiv 0$ or $u^{0}$ is a positive solution of $\left(E_{\infty}\right)$, and there exist $l$ bubbles $B^{i}=\left(B_{\alpha}^{i}\right)_{\alpha}, i=1 \ldots l$, such that, up to a subsequence,

$$
u_{\alpha}=u^{0}+\sum_{i=1}^{l} B_{\alpha}^{i}+S_{\alpha}
$$

where the sequence $\left(S_{\alpha}\right) \subset H_{1}^{p}(M)$ converges strongly to 0 in $H_{1}^{p}$, and

$$
I_{g}^{\alpha}\left(u_{\alpha}\right)=I_{g}^{\infty}\left(u^{0}\right)+\sum_{i=1}^{k} E\left(B^{i}\right)+o(1)
$$

where $I_{g}^{\alpha}$ and $I_{g}^{\infty}$ are the functional defined on $H_{1}^{p}(M)$ by (12) and (13) respectively, and the energy $E\left(B^{i}\right)$ of the bubble $B^{i}$ is defined by (5).

Moreover, there exists a constant $C>0$ independent of $\alpha$ and $x \in M$ such that for any $\alpha$ and any $x \in M$,

$$
\begin{gathered}
R_{\alpha}(x)^{\frac{n-k-p}{p}}\left|u_{\alpha}(x)-u^{0}(x)\right| \leq C, \text { and } \\
\lim _{R \rightarrow \infty} \lim _{\alpha \rightarrow+\infty} \sup _{x \in M \backslash \Omega_{\alpha}(R)} R_{\alpha}(x)^{\frac{n-k-p}{p}}\left|u_{\alpha}(x)-u^{0}(x)\right|=0
\end{gathered}
$$

where the $\left(x_{\alpha}^{i}\right)_{\alpha}$ and $\left(\mu_{\alpha}^{i}\right)_{\alpha}$ are the centers and the inverse of the weights of the bubble $B^{i}, R_{\alpha}(x)=\min _{i=1 \ldots l} d_{g}\left(G x_{\alpha}^{i}, G x\right)$ and, for $R>0, \Omega_{\alpha}(R)=\cup_{i=1}^{k} B_{G x_{\alpha}^{i}}\left(R \mu_{\alpha}^{i}\right)$. In the particular case where $p \leq 2, u^{0}=0$ and $u_{\alpha}$ is a solution of $\left(E_{\alpha}\right)$, we can prove that $\nabla f\left(x^{i}\right)=0$ for any $i$, where $x^{i}=\lim _{\alpha} x_{\alpha}^{i}$.

The paper is organized as follow. The first section is devoted to the proof of the $H_{1}^{p}$-decomposition, i.e. the relations (8) and (9) for a Palais-Smale sequence for the functional $I_{g}^{\alpha}$ defined by $(12)$, whereas the second one deals with the proof of the pointwise estimates (10) and (11).

\section{Proof of the $H_{1}^{p}$-decomposition for Palais-Smale sequences}

Let $I_{g}^{\alpha}$ be the functional defined on $H_{1}^{p}(M)$ by

$$
I_{g}^{\alpha}(u)=\frac{1}{p} \int_{M}|\nabla u|_{g}^{p} d v_{g}+\frac{1}{p} \int_{M} h_{\alpha}|u|^{p} d v_{g}-\frac{1}{p^{*}} \int_{M} f|u|^{p^{*}} d v_{g},
$$


and $\left(u_{\alpha}\right) \in H_{1, G}^{p}(M)$ be a Palais-Smale (P-S) sequence for $I_{g}^{\alpha}$ i.e. the sequence $\left(I_{g}^{\alpha}\left(u_{\alpha}\right)\right)$ is bounded and $D I_{g}^{\alpha}\left(u_{\alpha}\right) \rightarrow 0$ strongly in $H_{1}^{p}(M)^{\prime}$. We are going to prove that the relations (8) and (9) hold for $\left(u_{\alpha}\right)$ with generalized bubbles $B^{i}$. We will then prove that if the $u_{\alpha}$ are positive then the $B^{i}$ are bubbles.

It follows from Saintier [9] that the sequence $\left(u_{\alpha}\right)$ weakly converges, up to a subsequence, to a solution $u^{0} \in H_{1}^{p}(M)$ of the limit equation $\left(E_{\infty}\right)$. Since we can also assume that the convergence holds almost everywhere, we have $u^{0} \in$ $H_{1, G}^{p}(M)$. Let $v_{\alpha}=u_{\alpha}-u^{0} \in H_{1, G}^{p}(M)$. Then (cf Saintier [9]) $\left(v_{\alpha}\right)$ weakly converges to 0 in $H_{1}^{p}(M)$ and is a (P-S) sequence for the functional $I_{g}$ defined on $H_{1}^{p}(M)$ by

$$
I_{g}(u)=\frac{1}{p} \int_{M}|\nabla u|_{g}^{p} d v_{g}-\frac{1}{p^{*}} \int_{M} f|u|^{p^{*}} d v_{g}
$$

Moreover

$$
I_{g}\left(v_{\alpha}\right)=I_{g}^{\alpha}\left(u_{\alpha}\right)-I_{g}^{\infty}\left(u^{0}\right)+o(1)
$$

where $I_{g}^{\infty}$ is the functional defined on $H_{1}^{p}(M)$ by

$$
I_{g}^{\infty}(u)=\frac{1}{p} \int_{M}|\nabla u|_{g}^{p} d v_{g}+\frac{1}{p} \int_{M} h_{\infty}|u|^{p} d v_{g}-\frac{1}{p^{*}} \int_{M} f|u|^{p^{*}} d v_{g} .
$$

According to Faget [2], for any $\epsilon>0$, there exists a constant $C_{\epsilon}>0$ such that for any $u \in H_{1, G}^{p}(M)$,

$$
\left(\int_{M}|u|^{p^{*}} d v_{g}\right)^{\frac{p}{p^{*}}} \leq\left(\frac{K(n-k, p)^{p}}{A^{\frac{p}{n-k}}}+\epsilon\right) \int_{M}|\nabla u|_{g}^{p} d v_{g}+C_{\epsilon} \int_{M}|u|^{p} d v_{g}
$$

where $A$ denotes the minimal volume of $k$-dimensional $G$-orbits. We can then adapt Saintier ([9] step 1.4) to prove that if $\left(w_{\alpha}\right)$ is a (P-S) sequence for $I_{g}$ such that

$$
w_{\alpha} \rightarrow 0 \text { weakly in } H_{1}^{p}(M) \text { and } \lim _{\alpha} I_{g}\left(w_{\alpha}\right)<\|f\|_{\infty}^{-\frac{n-k-p}{p}} A \beta^{*},
$$

where $\beta^{*}=\frac{1}{(n-k) K(n-k, p)^{n-k}}$, then

$$
w_{\alpha} \rightarrow 0 \text { strongly in } H_{1}^{p} .
$$

Using this remark and the minoration (6) of the energy of a bubble, we can prove the theorem by induction by repeated use of the following lemma:

Lemma. Let $\left(v_{\alpha}\right)$ be a $(P-S)$ sequence for $I_{g}$ converging to 0 in $H_{1}^{p}$ weakly but not strongly. Then there exists a generalized bubble $B=\left(B_{\alpha}\right)$ such that $w_{\alpha}:=v_{\alpha}-B_{\alpha}$ is a $(P-S)$ sequence for $I_{g}$ weakly converging to 0 in $H_{1}^{p}$. Moreover

$$
I_{g}\left(w_{\alpha}\right)=I_{g}\left(v_{\alpha}\right)-E(B)+o(1) .
$$


The remainder of this section is devoted to the proof of this Lemma. The set of smooth $G$-invariant functions on $M$ being dense in $H_{1, G}^{p}(M)$ (see HebeyVaugon [6]), we can assume that the $v_{\alpha}$ 's are smooth. Independently, since the $v_{\alpha}$ 's don't converge strongly to 0 , the definition of a (P-S) sequence implies that

$$
\int_{M}\left|\nabla v_{\alpha}\right|_{g}^{p} d v_{g}=(n-k) \beta+o(1)
$$

and

$$
\int_{M} f\left|v_{\alpha}\right|^{p^{*}} d v_{g}=(n-k) \beta+o(1)
$$

for some $\beta \geq\|f\|_{\infty}^{-\frac{n-k-p}{p}} A \beta^{*}>0$. The compactness of $M$ then gives the existence of a point $x_{0} \in M$ such that for any $\delta>0$ small enough,

$$
\limsup _{\alpha \rightarrow+\infty} \int_{B_{G x_{0}}(\delta)} f\left|v_{\alpha}\right|^{p^{*}} d v_{g}>0 .
$$

The orbit $G x_{0}$ is called orbit of concentration. We give some preliminary properties of such an orbit:

Step 1.1 1) There are a finite number of concentration orbits. If $G x_{0}$ is one of them, then $\operatorname{dim} G x_{0}=k$ and $f\left(x_{0}\right)>0$. In the particular case where $p \leq 2$, $u^{0}=0$ and $u_{\alpha}$ is a solution of $\left(E_{\alpha}\right)$ for any $\alpha$, we also have $\nabla f\left(x_{0}\right)=0$. Moreover $G x_{0}$ is an orbit of concentration if and only if for any $\delta>0$,

$$
\limsup _{\alpha \rightarrow+\infty} \int_{B_{G x_{0}}(\delta)}\left|\nabla v_{\alpha}\right|_{g}^{p} d v_{g}>0 .
$$

2 ) Let $G x_{0}$ be an orbit of concentration for $\left(v_{\alpha}\right)$. According to 1) and in view of assumption $(\mathrm{H})$, there exist $\delta_{0}>0$ and a subgroup $G^{\prime}$ of $I \operatorname{som}_{g}(M)$ such that we can consider the Riemannian quotient $(n-k)$-manifold $\left(N:=B_{G x_{0}}\left(\delta_{0}\right) / G^{\prime}, \bar{g}\right)$. Then $\bar{x}_{0}$ is a point of concentration for $\left(\bar{v}_{\alpha}\right)$ in the sense that for any $\delta>0$ small,

$$
\limsup _{\alpha \rightarrow+\infty} \int_{B_{\bar{x}_{0}}^{\tilde{g}}(\delta)}\left|\nabla \bar{v}_{\alpha}\right|_{\tilde{g}}^{p} d v_{\tilde{g}}>0
$$

where $\tilde{g}$ is defined by $(2)$ and $\bar{v}_{\alpha}(\bar{x})=v_{\alpha}(x)$.

Proof. We first prove 1). Assume that $G x_{0}$ is an orbit of concentration of dimension $k^{\prime}>k$. Then there exists $\delta>0$ such that $\operatorname{dim} G x \geq k^{\prime}>k$ for any $x \in B_{G x_{0}}(\delta)$ (see Faget [3] lemma 2). It thus follows from Hebey-Vaugon (corollary 2 of [6]) and the inequality $\frac{\left(n-k^{\prime}\right) p}{n-k^{\prime}-p}>\frac{(n-k) p}{n-k-p}=p^{*}$ that the injection $H_{1, G}^{p}$ $\left(B_{G x_{0}}\left(\delta^{\prime}\right)\right) \hookrightarrow L^{p^{*}}\left(B_{G x_{0}}\left(\delta^{\prime}\right)\right)$ is compact for all $\delta^{\prime} \in(0, \delta)$. Since $v_{\alpha} \rightarrow 0$ weakly in $H_{1}^{p}(M)$, we get a contradiction with (16). Hence $G x_{0}$ is of minimal dimension $k$.

Since $\left(v_{\alpha}\right)$ is bounded in $H_{1, G}^{p}(M)$, there exist two finite positive $G$-invariant measures $\mu$ and $\nu$ such that $\left|v_{\alpha}\right|^{p^{*}} d v_{g} \rightarrow \nu$ and $\left|\nabla v_{\alpha}\right|_{g}^{p} d v_{g} \rightarrow \mu$ weakly in the sense 
of measure. Let $\epsilon>0$. According to Faget [2], there exists $C_{\epsilon}>0$ such that for any $\alpha$ and any $G$-invariant function $\phi \in C(M)$,

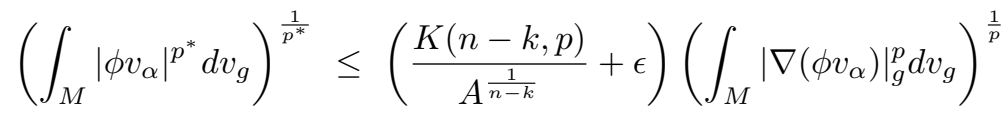

$$
\begin{aligned}
& +C_{\epsilon}\left(\int_{M}\left|\phi v_{\alpha}\right|^{p} d v_{g}\right)^{\frac{1}{p}} .
\end{aligned}
$$

Passing to the limit in $\alpha$ and then in $\epsilon$ in this inequality we get

$$
\left(\int_{M}|\phi|^{p^{*}} d \nu\right)^{\frac{1}{p^{*}}} \leq\left(\frac{K(n-k, p)}{A^{\frac{1}{n-k}}}\right)\left(\int_{M}|\phi|^{p} d \mu\right)^{\frac{1}{p}}
$$

for any $G$-invariant function $\phi \in C(M)$. Lemma 1.1 in Lions [7] then gives the existence of $I \subset \mathbb{N}$, a sequence of points $\left(x_{i}\right)_{i \in I} \subset M$ and two sequences of positive reals $\left(\mu_{i}\right)_{i \in I}$ and $\left(\nu_{i}\right)_{i \in I}$ such that

$$
\begin{aligned}
\left|v_{\alpha}\right|^{p^{*}} d v_{g} & \rightarrow \nu=\sum_{i \in I} \nu_{i} \delta_{G x_{i}}, \\
\left|\nabla v_{\alpha}\right|_{g}^{p} d v_{g} & \rightarrow \mu \geq \sum_{i \in I} \mu_{i} \delta_{G x_{i}}, \text { and } \\
\nu_{i}^{\frac{p}{p^{*}}} & \leq \frac{K(n-k, p)^{p}}{A^{\frac{p}{n-k}}} \mu_{i} \forall i \in I .
\end{aligned}
$$

where $\delta_{G x_{i}}$ is defined by $\delta_{G x_{i}}(\phi)=\int_{G} \phi\left(\sigma x_{i}\right) d m(\sigma)$ for $\phi \in C(M), m$ being the Haar measure of $G$ such that $m(G)=1$ (in particular, if $\phi$ is $G$-invariant, then $\left.\delta_{G x_{i}}(\phi)=\phi\left(x_{i}\right)\right)$. Let $\phi \in C(M)$. Then

$$
\begin{aligned}
o(1) & =D I_{g}\left(v_{\alpha}\right) \cdot\left(v_{\alpha} \phi\right) \\
& =\int_{M}\left|\nabla v_{\alpha}\right|_{g}^{p} \phi d v_{g}+\int_{M} v_{\alpha}\left|\nabla v_{\alpha}\right|^{p-2}\left(\nabla v_{\alpha}, \nabla \phi\right)_{g} d v_{g}-\int_{M} f \phi\left|v_{\alpha}\right|^{p^{*}} d v_{g} .
\end{aligned}
$$

By Hölder inequality, the second integral tends to 0 . We thus get by passing to the limit in the above expression that

$$
\int_{M} \phi d \mu=\int_{M} \phi f d \nu
$$

for any $\phi \in C(M)$. Hence $\mu=f \nu$. In particular $\mu\left(G x_{i}\right)=\int_{G x_{i}} f d \nu$ for any $i \in I$, and thus $\mu_{i} \leq f\left(x_{i}\right) \nu_{i}$ for any $i \in I$. This implies that $f\left(x_{i}\right)>0$ for any $i \in I$. Using (19), we obtain

and

$$
\nu_{i} \geq \frac{A K(n-k, p)^{k-n}}{f\left(x_{i}\right)^{\frac{n-k}{p}}} \geq \frac{A K(n-k, p)^{k-n}}{(\max f)^{\frac{n-k}{p}}},
$$

$$
\mu_{i} \geq \frac{A K(n-k, p)^{k-n}}{f\left(x_{i}\right)^{\frac{n-k-p}{p}}} \geq \frac{A K(n-k, p)^{k-n}}{(\max f)^{\frac{n-k-p}{p}}}
$$


for any $i \in I$. We now write using (15) that

$$
(n-k) \beta=\int_{M}\left|\nabla v_{\alpha}\right|_{g}^{p} d v_{g}+o(1)=\sum_{i \in I} \mu_{i} \geq \operatorname{card}(I) \frac{A K(n-k, p)^{k-n}}{(\max f)^{\frac{n-k-p}{p}}}
$$

which implies that $I$ is finite i.e. $\left(v_{\alpha}\right)$ has a finite number of orbits of concentration, namely the $G x_{i}, i \in I$. Eventually,

$$
\mu=f \nu=\sum_{i \in I} \nu_{i} f\left(x_{i}\right) \delta_{G x_{i}}
$$

which implies the equivalent definition (17) of an orbit of concentration.

We assume that $p \leq 2, u^{0} \equiv 0$ and $D I_{g}^{\alpha}\left(u_{\alpha}\right)=0$. Note that it follows from (19) and (20) that

$$
\begin{aligned}
\lim _{\alpha \rightarrow+\infty} \int_{M} \varphi\left|u_{\alpha}\right|^{p^{*}} d v_{g} & =\sum_{i \in I} \nu_{i} \varphi\left(x_{i}\right), \\
\lim _{\alpha \rightarrow+\infty} \int_{M} \varphi\left|\nabla u_{\alpha}\right|_{g}^{p} d v_{g} & =\sum_{i \in I} f\left(x_{i}\right) \nu_{i} \varphi\left(x_{i}\right)
\end{aligned}
$$

for all $\varphi \in C^{0}(M) G$-invariant.

We fix $i \in I$ and $G x_{i}$ an orbit of concentration. We consider the group $G^{\prime}$ given by the hypothesis $(\mathrm{H})$ taken at $x_{i}$ (note that an orbit of concentration has minimal dimension and therefore we can appply $(\mathrm{H}))$ and, given $\epsilon>0$, let $\eta \equiv \eta_{\bar{x}_{i}, \epsilon}$.

We assume that $\nabla f\left(x_{i}\right) \neq 0$. We consider a smooth $G^{\prime}$-invariant function $\phi$ with compact support in $B_{G x_{i}}(\delta)$ such that $\nabla \phi\left(x_{i}\right)=\nabla f\left(x_{i}\right)$ and $\nabla^{2} \phi\left(x_{i}\right)=0$. We let $\sigma:=|\nabla \phi|_{g}^{p-2} \nabla \phi$. Since $p \leq 2$, it follows from [12] that $u_{\alpha} \in H_{2}^{p}(M)$. In particular, the function $\left(\sigma, \nabla u_{\alpha}\right)_{g}$ belongs to $H_{1}^{p}(M)$. We let $\epsilon>0$ such that $\nabla \phi(x) \neq 0$ for all $x \in B_{G x_{i}}(\epsilon / 2)$ and we let $\eta$ defined above. With (21), we get that

$$
\frac{1}{p^{*}} \int_{M}(\sigma, \nabla f)_{g}\left|u_{\alpha}\right|^{p^{*}} d v_{g}=\frac{\nu_{i}}{p^{*}}\left|\nabla f\left(x_{i}\right)\right|_{g}^{p}\left(x_{i}\right)+o(1)
$$

Independently, we have that

$$
\begin{aligned}
\frac{1}{p^{*}} \int_{M}(\sigma, \nabla f)_{g}\left|u_{\alpha}\right|^{p^{*}} d v_{g}=\frac{1}{p^{*}} \int_{M} \eta(\sigma, \nabla f)_{g}\left|u_{\alpha}\right|^{p^{*}} d v_{g}+o(1) \\
=\frac{-1}{p^{*}} \int_{M} \operatorname{div}\left(\eta\left|u_{\alpha}\right|^{p^{*}}|\nabla \phi|_{g}^{p-2} \nabla \phi\right) f d v_{g}+o(1) \\
=\frac{1}{p^{*}} \int_{M} \eta f\left|u_{\alpha}\right|^{p^{*}} \Delta_{p} \phi d v_{g}-\frac{1}{p^{*}} \int_{M}|\nabla \phi|^{p-2}(\nabla \phi, \nabla \eta)_{g}\left|u_{\alpha}\right|^{p^{*}} f d v_{g} \\
\quad-\int_{M} \eta f\left|u_{\alpha}\right|^{p^{*}-2} u_{\alpha}\left(\sigma, \nabla u_{\alpha}\right)_{g} d v_{g}+o(1) \\
=\frac{\nu_{i}}{p^{*}} \eta\left(x_{i}\right) f\left(x_{i}\right)_{\Delta_{p}} \phi\left(x_{i}\right)-\int_{M}\left|\nabla u_{\alpha}\right|_{g}^{p-2}\left(\nabla u_{\alpha}, \nabla\left(\eta\left(\sigma, \nabla u_{\alpha}\right)_{g}\right)\right)_{g} d v_{g} \\
\quad-\int_{M} h_{\alpha}\left|u_{\alpha}\right|^{p-2} u_{\alpha} \eta\left(\sigma, \nabla u_{\alpha}\right)_{g} d v_{g}+o(1)
\end{aligned}
$$


Thanks to Hölder's inequality, the last integral goes to 0 , and straightforward computations yield that $\Delta_{p} \phi\left(x_{i}\right)=0$. We then get that

$$
\frac{\nu_{i}}{p^{*}}\left|\nabla f\left(x_{i}\right)\right|_{g}^{p}\left(x_{i}\right)=-\int_{M}\left|\nabla u_{\alpha}\right|_{g}^{p-2}\left(\nabla u_{\alpha}, \nabla\left(\eta\left(\sigma, \nabla u_{\alpha}\right)_{g}\right)\right)_{g} d v_{g}+o(1) .
$$

Passing to the quotient manifolds $N:=B_{G^{\prime} x_{i}}(\delta) / G^{\prime}$ and using the $G^{\prime}$-invariance, we get that

$$
\frac{\nu_{i}}{p^{*}}\left|\nabla f\left(x_{i}\right)\right|_{g}^{p}\left(x_{i}\right)=-\int_{N}\left|\nabla \bar{u}_{\alpha}\right|_{\bar{g}}^{p-2}\left(\nabla \bar{u}_{\alpha}, \nabla\left(\bar{\eta}\left(\bar{\sigma}, \nabla \bar{u}_{\alpha}\right) \bar{g}\right)\right) \overline{\bar{g}} \bar{d} d v_{\bar{g}}+o(1),
$$

where we have that $\bar{\eta} \circ \Pi=\eta, \bar{u}_{\alpha} \circ \Pi=u_{\alpha}$ and $\bar{\sigma} \circ \Pi=\sigma$. We have that

$$
\begin{aligned}
\left|\nabla \bar{u}_{\alpha}\right|_{\bar{g}}^{p-2}\left(\nabla \bar{u}_{\alpha}, \nabla\left(\bar{\eta}\left(\bar{\sigma}, \nabla \bar{u}_{\alpha}\right)_{\bar{g}}\right)\right)_{\bar{g}}= & \frac{1}{p} \bar{\eta}\left(\bar{\sigma}, \nabla\left|\nabla \bar{u}_{\alpha}\right| \frac{p}{\bar{g}}\right)_{g} \\
& +\left|\nabla \bar{u}_{\alpha}\right|_{\bar{g}}^{p-2} \nabla^{i} \bar{u}_{\alpha} \nabla^{j} \bar{u}_{\alpha} \nabla_{j}\left(\bar{\eta} \bar{\sigma}_{j}\right) .
\end{aligned}
$$

Using Cartan's expansion of $\bar{g}$ in the exponential chart at $\bar{x}_{i}$ and noting that $\nabla \sigma\left(x_{i}\right)=0$ (here, one uses that $\nabla^{2} \phi\left(x_{i}\right)=0$ ), we get that

$$
\left|\nabla \bar{u}_{\alpha}\right| \frac{p-2}{\bar{g}}\left(\nabla \bar{u}_{\alpha}, \nabla\left(\bar{\eta}\left(\bar{\sigma}, \nabla \bar{u}_{\alpha}\right)_{\bar{g}}\right)\right)_{\bar{g}}=\frac{1}{p} \bar{\eta}\left(\bar{\sigma}, \nabla\left|\nabla \bar{u}_{\alpha}\right| \frac{p}{\bar{g}}\right)_{\bar{g}}+O\left(d_{\bar{g}}\left(\bar{x}_{i}, \bar{x}\right)\left|\nabla \bar{u}_{\alpha}\right| \frac{p}{\bar{g}}\right) .
$$

With (22), we get that

$$
\int_{N} d_{\bar{g}}\left(\bar{x}_{i}, \bar{x}\right)\left|\nabla \bar{u}_{\alpha}\right|_{\bar{g}}^{p}=o(1)
$$

when $\alpha \rightarrow+\infty$. We then get that

$$
\begin{aligned}
\frac{\nu_{i}}{p^{*}}\left|\nabla f\left(x_{i}\right)\right|_{g}^{p}\left(x_{i}\right) & =-\frac{1}{p} \int_{N} \bar{\eta}\left(\bar{\sigma}, \nabla\left|\nabla \bar{u}_{\alpha}\right|_{\bar{g}}^{p}\right)_{\bar{g}} \bar{v} d v_{\bar{g}}+o(1) \\
& =-\frac{1}{p} \int_{M} \eta\left(\sigma, \nabla\left|\nabla u_{\alpha}\right|_{g}^{p}\right)_{g} d v_{g}+o(1) \\
& =-\frac{1}{p} \int_{M}\left(\eta \Delta_{p} \phi-|\nabla \phi|_{g}^{p-2}(\nabla \eta, \nabla \phi)_{g}\right)\left|\nabla u_{\alpha}\right|_{g}^{p} d v_{g}+o(1) \\
& =-\frac{1}{p} \Delta_{p} \phi\left(x_{i}\right) f\left(x_{i}\right) \nu_{i}+o(1)=o(1)
\end{aligned}
$$

since $\nabla^{2} \phi\left(x_{i}\right)=0$. We then get that $\nabla f\left(x_{i}\right)=0$, a contradiction with our initial hypothesis. Then $\nabla f\left(x_{i}\right)=0$ for all $i \in I$. This proof requires the use of $\left(\sigma, \nabla u_{\alpha}\right)_{g}$ as a test-function and thus cannot work if we deal with (P-S) sequence since then we need the $u_{\alpha}$ to be bounded in $H_{2}^{p}$ (which is irrevelant here because it implies the strong convergence in $H_{1}^{p}$ of the $u_{\alpha}$ to $\left.u^{0}\right)$. This explains the restriction imposed to get $\nabla f\left(x_{i}\right)=0$. 
We now prove 2). According to 1$), \operatorname{dim} G x_{0}=k$. Assumption (H) then gives $\delta_{0}>0$ and a subgroup $G^{\prime}$ of $\operatorname{Isom}_{g}(M)$ such that (H1) and (H2) are satisfied. We have for $\delta \in\left(0, \delta_{0}\right)$

$$
\int_{B_{G x_{0}}(\delta)}\left|\nabla v_{\alpha}\right|_{g}^{p} d v_{g}=\int_{B_{\bar{x}_{0}}^{\bar{g}}(\delta)}\left|\nabla \bar{v}_{\alpha}\right|_{\bar{g}}^{p} \bar{v} d v_{\bar{g}}=\int_{B_{\bar{x}_{0}}^{\bar{g}}(\delta)}\left|\nabla \bar{v}_{\alpha}\right|_{\tilde{g}}^{p} d v_{\tilde{g}},
$$

where $\bar{v}_{\alpha} \circ \Pi=v_{\alpha}, \Pi: B_{G x_{0}}(\delta) \rightarrow N$ being the canonical surjection. Let

$$
m=\inf _{\bar{x} \in B_{\bar{x}_{0}}^{\bar{g}}(\delta)} \bar{v}(\bar{x})^{\frac{1}{n-k-p}} \text { and } M=\sup _{\bar{x} \in B_{\bar{x}_{0}}^{\bar{g}}(\delta)} \bar{v}(\bar{x})^{\frac{1}{n-k-p}} .
$$

Then for any $\bar{x} \in B_{\bar{x}_{0}}^{\bar{g}}(\delta)$,

$$
\frac{1}{M} d_{\bar{g}}\left(\bar{x}, \bar{x}_{0}\right) \leq d_{\tilde{g}}\left(\bar{x}, \bar{x}_{0}\right) \leq \frac{1}{m} d_{\bar{g}}\left(\bar{x}, \bar{x}_{0}\right) .
$$

Hence

$$
\int_{B_{G x_{0}}(\delta)}\left|\nabla v_{\alpha}\right|^{p} d v_{g} \leq \int_{B_{\bar{x}_{0}\left(\frac{\delta}{m}\right)}^{\tilde{g}}}\left|\nabla \bar{v}_{\alpha}\right|_{\tilde{g}}^{p} d v_{\tilde{g}}
$$

which proves the claim.

Step 1.2 Let $G x_{0}$ be an orbit such that there exist $\delta_{0}>0$ and a subgroup $G^{\prime} \subset \operatorname{Isom}_{g}(M)$ satisfying $(\mathrm{H} 1)$ and $(\mathrm{H} 2)$. Then $\left(\bar{v}_{\alpha}\right)$ is a (P-S) sequence for the functional $\bar{I}_{\tilde{g}}$ defined on $\stackrel{\circ}{H_{1}^{p}}(N)$ by

$$
\bar{I}_{\tilde{g}}(\bar{u})=\frac{1}{p} \int_{N}|\nabla \bar{u}|_{\tilde{g}}^{p} d v_{\tilde{g}}-\frac{1}{p^{*}} \int_{N} \bar{f}|\bar{u}|^{p^{*}} \bar{v}^{-\frac{p}{n-k-p}} d v_{\tilde{g}}
$$

where $N=B_{G x_{0}}\left(\delta_{0}\right) / G^{\prime}, \bar{f} \circ \Pi=f$ and $\Pi: B_{G x_{0}}\left(\delta_{0}\right) \rightarrow N$ is the canonical surjection.

Proof. Let $\bar{\phi} \in C_{c}^{\infty}(N)$ and $\phi \in C_{c}^{\infty}\left(B_{G x_{0}}\left(\delta_{0}\right)\right)$ such that $\bar{\phi} \circ \Pi=\phi$. Then

$$
\begin{aligned}
& o(1)\|\bar{\phi}\|_{H_{1}^{p}(N)}=o(1)\|\phi\|_{H_{1}^{p}(M)}=D I_{g}\left(v_{\alpha}\right) \phi \\
& =\int_{B_{G x_{0}}\left(\delta_{0}\right)}\left|\nabla v_{\alpha}\right|_{g}^{p-2}\left(\nabla v_{\alpha}, \nabla \phi\right)_{g} d v_{g}-\int_{B_{G x_{0}}\left(\delta_{0}\right)} f\left|v_{\alpha}\right|^{p^{*}-2} v_{\alpha} \phi d v_{g} \\
& =\int_{B_{\bar{x}_{0}}^{\bar{g}}\left(\delta_{0}\right)}\left|\nabla \bar{v}_{\alpha}\right|_{\bar{g}}^{p-2}\left(\nabla \bar{v}_{\alpha}, \nabla \bar{\phi}\right)_{\bar{g}} \bar{v} d v_{\bar{g}}-\int_{B_{\bar{x}_{0}}^{\bar{g}}\left(\delta_{0}\right)} \bar{f}\left|\bar{v}_{\alpha}\right|^{p^{*}-2} \bar{v}_{\alpha} \bar{\phi} \bar{v} d v_{\bar{g}} \\
& =\int_{B_{\bar{x}_{0}}^{\bar{g}}\left(\delta_{0}\right)}\left|\nabla \bar{v}_{\alpha}\right|_{\tilde{g}}^{p-2}\left(\nabla \bar{v}_{\alpha}, \nabla \bar{\phi}\right)_{\tilde{g}} d v_{\tilde{g}} \\
& -\int_{B_{\bar{x}_{0}}^{\bar{g}}\left(\delta_{0}\right)} \bar{f}\left|\bar{v}_{\alpha}\right|^{p^{*}-2} \bar{v}_{\alpha} \bar{\phi} \bar{v}^{-\frac{p}{n-k-p}} d v_{\tilde{g}} \\
& =D \bar{I}_{\tilde{g}}\left(\bar{v}_{\alpha}\right) . \bar{\phi}
\end{aligned}
$$


As explained above, there exists an orbit of concentration $G x_{0}$. According to Step 1.1, $\operatorname{dim} G x_{0}=k$. Assumption $(\mathrm{H})$ then gives $\delta_{0}>0$ and a subgroup $G^{\prime} \subset \operatorname{Isom}_{g}(M)$ satisfying $(\mathrm{H} 1)$ and $(\mathrm{H} 2)$ on $B_{G x_{0}}\left(2 \delta_{0}\right)$. We let $N=B_{G x_{0}}\left(\delta_{0}\right) / G^{\prime}$ and consider, for $t>0$,

$$
\mu_{\alpha}(t)=\max _{\bar{x} \in N} \int_{B_{\bar{x}}^{\tilde{g}}(t)}\left|\nabla \bar{v}_{\alpha}\right|_{\tilde{g}}^{p} d v_{\tilde{g}} .
$$

In view of Step 1.1, there exist $\lambda_{0}$ such that, up to a subsequence, for any $\alpha$

$$
\mu_{\alpha}\left(\delta_{0}\right) \geq \int_{B_{\bar{x}_{0}}^{\tilde{g}}\left(\delta_{0}\right)}\left|\nabla \bar{v}_{\alpha}\right|_{\tilde{g}}^{p} d v_{\tilde{g}} \geq \lambda_{0} .
$$

Since $\mu_{\alpha}$ is continuous, we then get for any $\lambda \in\left(0, \lambda_{0}\right)$ the existence of $t_{\alpha} \in\left(0, \delta_{0}\right)$ and $\bar{x}_{\alpha} \in N, \bar{x}_{\alpha} \rightarrow \bar{x}_{0}$, such that for any $\alpha$

$$
\mu_{\alpha}\left(t_{\alpha}\right)=\int_{B_{\bar{x}_{\alpha}}^{\tilde{g}}\left(t_{\alpha}\right)}\left|\nabla \bar{v}_{\alpha}\right|_{\tilde{g}}^{p} d v_{\tilde{g}}=\lambda .
$$

In view of to Step 1.2, $\left(\bar{v}_{\alpha}\right)$ is a $(\mathrm{P}-\mathrm{S})$ sequence for $\bar{I}_{\tilde{g}}$ on $\stackrel{\circ}{H_{1}^{p}}(N)$. According to Saintier [9], there exist a sequence $R_{\alpha} \rightarrow+\infty$ and $v \in D_{1}^{p}\left(\mathbb{R}^{n-k}\right)$, (where $D_{1}^{p}\left(\mathbb{R}^{n-k}\right)$ is the completion of $C_{c}^{\infty}\left(\mathbb{R}^{n-k}\right)$ for the norm $\left.u \mapsto\|\nabla u\|_{p}\right)$ such that

$$
\tilde{v}_{\alpha} \rightarrow v \text { in } H_{1, l o c}^{p}\left(\mathbb{R}^{n-k}\right)
$$

and $v \not \equiv 0$, where, if $i_{\tilde{g}}\left(\bar{x}_{0}\right)$ denotes the injectivity radius of $(N, \tilde{g})$ at $\bar{x}_{0}$,

$$
\tilde{v}_{\alpha}(x)=R_{\alpha}^{-\frac{n-k-p}{p}} \bar{v}_{\alpha}\left(\exp _{\bar{x}_{\alpha}}\left(R_{\alpha}^{-1} x\right)\right), x \in B_{0}\left(R_{\alpha} i_{\tilde{g}}\left(\bar{x}_{0}\right)\right) .
$$

Actually, the analysis in [9] is performed with a constant function in front of $|\bar{u}|^{p^{*}}$ in the functional $\bar{I}_{\tilde{g}}(\bar{u})$. In our context here, $\bar{f} \bar{v}^{-\frac{p}{n-k-p}}$ is not constant: however, the analysis for the proof of the result above works the same.

We now prove that

Step $1.3 v$ is a solution of the Euclidean equation

$$
\begin{aligned}
\Delta_{p, \xi} v & =\bar{f}\left(\bar{x}_{0}\right) \bar{v}\left(\bar{x}_{0}\right)^{-\frac{p}{n-k-p}}|v|^{p^{*}-2} v \\
& =f\left(x_{0}\right) \operatorname{Vol}\left(G x_{0}\right)^{-\frac{p}{n-k-p}}|v|^{p^{*}-2} v
\end{aligned}
$$

Proof. Let $\phi \in C_{c}^{\infty}\left(\mathbb{R}^{n-k}\right)$ and $R>0$ such that supp $\phi \subset B_{0}(R)$. For $\alpha$ large enough, we define $\phi_{\alpha} \in C_{c}^{\infty}(N)$ by

$$
\phi_{\alpha}(\bar{x})=R_{\alpha}^{\frac{n-k-p}{p}} \phi\left(R_{\alpha} \exp _{\bar{x}_{\alpha}}(\bar{x})\right) .
$$


Then $\left(\phi_{\alpha}\right)$ is bounded in $\stackrel{\circ}{H_{1}^{p}}(N)$. Thus

$$
\begin{aligned}
o(1)= & D \bar{I}_{\tilde{g}}\left(\bar{v}_{\alpha}\right) \phi_{\alpha} \\
= & \int_{B_{0}(R)}\left|\nabla \tilde{v}_{\alpha}\right|_{\tilde{g}_{\alpha}}^{p-2}\left(\nabla \tilde{v}_{\alpha}, \nabla \phi\right)_{\tilde{g}_{\alpha}} d v_{\tilde{g}_{\alpha}} \\
& -\int_{B_{0}(R)}\left|\tilde{v}_{\alpha}\right|^{p^{*}-2} \tilde{v}_{\alpha} \phi \bar{v}\left(\exp _{\bar{x}_{\alpha}}\left(R_{\alpha}^{-1} x\right)\right)^{-\frac{p}{n-k-p}} \bar{f}\left(\exp _{\bar{x}_{\alpha}}\left(R_{\alpha}^{-1} x\right)\right) d v_{\tilde{g}_{\alpha}}
\end{aligned}
$$

where $\tilde{g}_{\alpha}$ is the metric defined in the Euclidean ball $B_{0}\left(i_{\tilde{g}} R_{\alpha}\right) \subset \mathbb{R}^{n-k}$ by

$$
\tilde{g}_{\alpha}(x)=\left(\exp _{\bar{x}_{\alpha}}^{*} \tilde{g}\right)\left(R_{\alpha}^{-1} x\right) .
$$

Since $R_{\alpha} \rightarrow+\infty$, the $\tilde{g}_{\alpha}$ converge locally uniformly to the Euclidean metric $\xi$. Passing to the limit, we then get using (23) that

$$
\int_{\mathbb{R}^{n-k}}|\nabla v|_{\xi}^{p-2}(\nabla v, \nabla \phi)_{\xi} d x-\bar{f}\left(\bar{x}_{0}\right) \bar{v}\left(\bar{x}_{0}\right)^{-\frac{p}{n-k-p}} \int_{\mathbb{R}^{n-k}}|v|^{p^{*}-2} v \phi d x=0
$$

which proves Step 1.3.

For $\delta>0$ small, we let

$$
\bar{B}_{\alpha}(\bar{x})=\eta_{\bar{x}_{\alpha}, \delta}(\bar{x}) R_{\alpha}^{\frac{n-k-p}{p}} v\left(R_{\alpha} \exp _{\bar{x}_{\alpha}}^{-1}(\bar{x})\right)
$$

and $\bar{w}_{\alpha}=\bar{v}_{\alpha}-\bar{B}_{\alpha}$. Then, according to Saintier ([9] Step 2.4),

$$
\begin{gathered}
\bar{B}_{\alpha} \rightarrow 0 \text { weakly in } \stackrel{\circ}{H_{1}^{p}}(N), \\
D \bar{I}_{\tilde{g}}\left(\bar{B}_{\alpha}\right) \rightarrow 0 \text { and } D \bar{I}_{\tilde{g}}\left(\bar{w}_{\alpha}\right) \rightarrow 0 \text { strongly in } \stackrel{\circ}{H_{1}^{p}}(N)^{\prime}, \\
\bar{I}_{\tilde{g}}\left(\bar{w}_{\alpha}\right)=\bar{I}_{\tilde{g}}\left(\bar{v}_{\alpha}\right)-E(v)+o(1)
\end{gathered}
$$

where

$$
E(v)=\frac{1}{p} \int_{\mathbb{R}^{n-k}}|\nabla v|_{\xi}^{p} d x-\frac{\bar{v}\left(\bar{x}_{0}\right)^{-\frac{p}{n-k-p}} f\left(x_{0}\right)}{p^{*}} \int_{\mathbb{R}^{n-k}}|v|^{p^{*}} d x .
$$

We now define a bubble $\left(B_{\alpha}\right)$ by the relation

$$
B_{\alpha}=\bar{B}_{\alpha} \circ \Pi
$$

and $w_{\alpha}=v_{\alpha}-B_{\alpha}$. We now claim that the following holds: 


\section{Step 1.4}

$$
\begin{gathered}
w_{\alpha} \rightarrow 0 \text { weakly in } H_{1}^{p}(M), \\
D I_{g}\left(B_{\alpha}\right) \rightarrow 0 \text { and } D I_{g}\left(w_{\alpha}\right) \rightarrow 0, \\
I_{g}\left(w_{\alpha}\right)=I_{g}\left(v_{\alpha}\right)-E(v)+o(1) .
\end{gathered}
$$

Proof. We first prove that $B_{\alpha} \rightarrow 0$ weakly in $H_{1}^{p}$ (which implies (27) since $v_{\alpha} \rightarrow 0$ weakly in $\left.H_{1}^{p}\right)$. Since $\left(B_{\alpha}\right) \subset H_{1, G^{\prime}}^{p}(M)$ is bounded in $H_{1}^{p}$, it suffices to prove that $B_{\alpha} \rightarrow 0$ weakly in $L_{G^{\prime}}^{p}(M)$. Let $\psi \in L_{G^{\prime}}^{q}(M), q=\frac{p}{p-1}$, and $\bar{\psi} \in L^{q}(N, \tilde{g})$ be such that $\psi=\bar{\psi} \circ \Pi$ in $B_{G x_{0}}(2 \delta)$. Then, using (24),

$$
\int_{M} B_{\alpha} \psi d v_{g}=\int_{N} \bar{B}_{\alpha} \bar{\psi} \bar{v}^{-\frac{p}{n-k-p}} d v_{\tilde{g}} \rightarrow 0 .
$$

We prove in the same way that $D I_{g}\left(B_{\alpha}\right) \rightarrow 0$. We now prove that

$$
D I_{g}\left(w_{\alpha}\right) \rightarrow 0
$$

Let $\phi \in H_{1, G}^{p}(M), \delta \in\left(0, \delta_{0} / 6\right)$ and $\eta_{0} \equiv \eta_{\bar{x}_{0}, 3 \delta} \in C_{c}^{\infty}\left(B_{G x_{0}}(6 \delta)\right)$. For $\alpha$ large enough so that $d_{\bar{g}}\left(\bar{x}_{\alpha}, \bar{x}_{0}\right)<\delta$ (in particular supp $\bar{B}_{\alpha} \subset B_{\bar{x}_{\alpha}}(2 \delta) \subset B_{\bar{x}_{0}}(3 \delta)$ ), straightforward computations yield

$$
\begin{aligned}
D I_{g}\left(w_{\alpha}\right) \phi & =D I_{g}\left(w_{\alpha}\right)\left(\eta_{0} \phi\right)+D I_{g}\left(w_{\alpha}\right)\left(\left(1-\eta_{0}\right) \phi\right) \\
& =D \bar{I}_{\tilde{g}}\left(\bar{w}_{\alpha}\right)\left(\overline{\eta_{0} \phi}\right)+D I_{g}\left(v_{\alpha}\right)\left(\left(1-\eta_{0}\right) \phi\right) \\
& =o\left(\left\|\overline{\eta_{0} \phi}\right\|_{H_{1}^{p}(N)}\right)+o\left(\left\|\left(1-\eta_{0}\right) \phi\right\|_{H_{1}^{p}(M)}\right) \\
& =o\left(\|\phi\|_{H_{1}^{p}(M)}\right)
\end{aligned}
$$

Now consider $\phi \in H_{1}^{p}(M)$ et $\phi_{G} \in H_{1, G}^{p}(M)$ defined by

$$
\phi_{G}(x)=\int_{G} \phi(\sigma x) d m(\sigma)
$$

where $m$ is the Haar measure of $\mathrm{G}$ such that $m(G)=1$. Then, according to what we just did,

$$
D I_{g}\left(w_{\alpha}\right) \phi_{G}=o(1)\left\|\phi_{G}\right\|_{H_{1}^{p}}
$$

with

$$
\begin{aligned}
D I_{g}\left(w_{\alpha}\right) \phi_{G}= & \int_{G}\left(\int_{M}\left|\nabla w_{\alpha}\right|^{p-2}\left(\nabla w_{\alpha}, \nabla(\phi \circ \sigma)\right)_{g} d v_{g}\right) d m(\sigma) \\
& -\int_{G}\left(\int_{M} f\left|w_{\alpha}\right|^{p^{*}-2} w_{\alpha}(\phi \circ \sigma) d v_{g}\right) d m(\sigma) \\
= & m(G) D I_{g}\left(w_{\alpha}\right) \phi
\end{aligned}
$$


and, using Hölder inequality,

$$
\begin{aligned}
\left\|\phi_{G}\right\|_{H_{1}^{p}}^{p}= & \int_{M}\left|\int_{G} \nabla(\phi \circ \sigma) d m(\sigma)\right|^{p} d v_{g}+\int_{M}\left|\int_{G}(\phi \circ \sigma) d m(\sigma)\right|^{p} d v_{g} \\
\leq & m(G)^{p-1} \int_{M}\left(\int_{G}|\nabla(\phi \circ \sigma)|^{p} d m(\sigma)\right) d v_{g} \\
& +m(G)^{p-1} \int_{M}\left(\int_{G}|\phi \circ \sigma|^{p} d m(\sigma)\right) d v_{g} \\
\leq & \|\phi\|_{H_{1}^{p}}^{p} .
\end{aligned}
$$

Hence

$$
D I_{g}\left(w_{\alpha}\right) \phi=o(1)\|\phi\|_{H_{1}^{p}}
$$

It remains to prove $(29)$. We write that

$$
I_{g}\left(w_{\alpha}\right)=\frac{1}{p} \int_{M \backslash B_{G x_{0}}(2 \delta)}\left|\nabla v_{\alpha}\right|_{g}^{p} d v_{g}-\frac{1}{p^{*}} \int_{M \backslash B_{G x_{0}}(2 \delta)} f\left|v_{\alpha}\right|^{p^{*}} d v_{g}+\bar{I}_{\tilde{g}}\left(\bar{w}_{\alpha}\right) .
$$

We then get using (26) that

$$
\begin{aligned}
I_{g}\left(w_{\alpha}\right)= & \frac{1}{p} \int_{M \backslash B_{G x_{0}}(2 \delta)}\left|\nabla v_{\alpha}\right|_{g}^{p} d v_{g}-\frac{1}{p^{*}} \int_{M \backslash B_{G x_{0}}(2 \delta)} f\left|v_{\alpha}\right|^{p^{*}} d v_{g}+\bar{I}_{\tilde{g}}\left(\bar{v}_{\alpha}\right) \\
& -E(v)+o(1) \\
= & I_{g}\left(v_{\alpha}\right)-E(v)+o(1)
\end{aligned}
$$

which proves (29). Note that $v \not \equiv 0$.

This ends the proof of the Lemma and thus of the $H_{1}^{p}$-decomposition for a Palais-Smale sequences $\left(u_{\alpha}\right)$ for $I_{g}^{\alpha}$ of arbitrary sign. If we assume that $u_{\alpha}>0$ for any $\alpha$, then $u^{0} \geq 0$ a.e. since $u_{\alpha} \rightarrow u^{0}$ weakly in $H_{1}^{p}$ and thus also almost everywhere (up to a subsequence). Since $u^{0} \in H_{1}^{p}(M)$ is a weak solution to $\left(E_{\infty}\right)$, it follows from Tolksdorf [12] that $u^{0} \in C^{1, \theta}(M)$ for some $\theta \in(0,1)$. We then deduce from Vazquez' maximum principle [13] that $u_{0} \equiv 0$ or $u^{0}>0$ everywhere. Moreover, according to Saintier [9], the $\bar{B}^{i}$ are bubbles and hence so are the $B^{i}$, $1 \leq i \leq k$.

\section{Proof of the $C^{0}$-estimates (10) and (11)}

Let $\left(u_{\alpha}\right)$ be a bounded sequence of positive solutions of $\left(E_{\alpha}\right)$. We prove in this section the pointwise estimates of Theorem 0.1. We first prove (10). By standard regularity results (see Tolksdorff [12]), we know that $u^{0} \in C^{1, \theta}(M)$ for some $\theta \in(0,1)$, where $u^{0}$ is the weak limit in $H_{1}^{p}$ of the $u_{\alpha}$ 's. It thus suffices to prove that there exists $C>0$ such that for every $\alpha$ and every $x \in M$,

$$
R_{\alpha}(x)^{\frac{n-k-p}{p}} u_{\alpha}(x) \leq C .
$$


Actually, we are going to prove the following stronger result: there exists $C>0$ such that

$$
v_{\alpha}(x):=R_{\alpha}^{\prime}(x)^{\frac{n-k-p}{p}} u_{\alpha}(x) \leq C
$$

for all $x \in M$ and all $\alpha>0$, where

$$
R_{\alpha}^{\prime}(x)=\min _{i=1, \ldots, l} d_{g}\left(G_{i}^{\prime} x, G_{i}^{\prime} x_{\alpha}^{i}\right)
$$

and for all $i \in\{1, \ldots, l\}$, the group $G_{i}^{\prime}$ is given by hypothesis (H) at the orbit of concentration $G x_{\infty}^{i}$, where $\lim _{\alpha \rightarrow+\infty} x_{\alpha}^{i}=x_{\infty}^{i}$.

We assume by contradiction that there exists $y_{\alpha} \in M$ such that

$$
v_{\alpha}\left(y_{\alpha}\right)=\max _{x \in M} v_{\alpha}(x) \rightarrow+\infty
$$

when $\alpha \rightarrow+\infty$ and we let $\mu_{\alpha}:=u_{\alpha}\left(y_{\alpha}\right)^{-p /(n-k-p)} \rightarrow 0$ when $\alpha \rightarrow+\infty$. We let $\lim _{\alpha \rightarrow+\infty} y_{\alpha}=y_{0}$, up to extraction.

We claim that the orbit $G y_{0}$ has minimal dimension $k$. Indeed, we argue by contradiction and assume that $\operatorname{dim} G y_{0}>k$. As in Step 1.1, we then get that there exist $q_{0}>p^{*}$ and $\delta>0$ such that $\lim _{\alpha \rightarrow+\infty} u_{\alpha}=u^{0}$ in $L^{q_{0}}\left(B_{G y_{0}}(\delta)\right)$. It then follows from $\left(E_{\alpha}\right)$ and standard regularity theory that $\lim _{\alpha \rightarrow+\infty} u_{\alpha}=u^{0}$ in $C^{0}\left(B_{G y_{0}}\left(\delta^{\prime}\right)\right)$ for all $\delta^{\prime}<\delta$. A contradiction with the assumption (32). This proves the claim.

We then let $G^{\prime}$ be the group given by hypothesis $(H)$ at the point $y_{0}$. We let $I_{0}=\left\{i \in\{1, \ldots, l\} / x_{\infty}^{i} \in G y_{0}\right\}$ (note that $I_{0}$ may be empty). Then, for all $i \in I_{0}$, we have that $G^{\prime}=G_{i}^{\prime}$. We consider the quotient manifold $N:=B_{G^{\prime} y_{0}}(\delta) / G^{\prime}$, where $\delta>0$ is small and given by $(\mathrm{H})$. Here again, we consider the function $\bar{u}_{\alpha}(\bar{x})=u_{\alpha}(x)$ for $\bar{x} \in N$. We fix $R_{0} \in\left(0, i_{\bar{g}}\left(\bar{y}_{0}\right)\right)$ and we consider the function $w_{\alpha}$ defined on the Euclidean ball $B_{0}\left(R_{0} \mu_{\alpha}^{-1}\right)$ by

$$
w_{\alpha}(x):=\mu_{\alpha}^{\frac{n-k-p}{p}} \bar{u}_{\alpha}\left(\exp _{\bar{y}_{\alpha}}\left(\mu_{\alpha} x\right)\right) .
$$

In this expression, the exponential map is taken wrt the metric $\bar{g}$. For $\rho>0$ and $x \in B_{0}(\rho) \subset \mathbb{R}^{n-k}$, we let $z_{\alpha} \in M$ be such that $G^{\prime} z_{\alpha}=\bar{z}_{\alpha}=\exp _{\bar{y}_{\alpha}}\left(\mu_{\alpha} x\right)$. Given $i \in I_{0}$, we get that

$$
\begin{aligned}
d_{g}\left(G^{\prime} z_{\alpha}, G^{\prime} x_{\alpha}^{i}\right) & \geq d_{g}\left(G^{\prime} x_{\alpha}^{i}, G^{\prime} y_{\alpha}\right)-d_{g}\left(G^{\prime} y_{\alpha}, G^{\prime} z_{\alpha}\right) \\
& \geq R_{\alpha}^{\prime}\left(y_{\alpha}\right)-d_{\bar{g}}\left(\bar{y}_{\alpha}, \bar{z}_{\alpha}\right) \\
& \geq R_{\alpha}^{\prime}\left(y_{\alpha}\right)-\mu_{\alpha}|x| \\
& \geq\left(1-\frac{\rho \mu_{\alpha}}{R_{\alpha}^{\prime}\left(y_{\alpha}\right)}\right) R_{\alpha}^{\prime}\left(y_{\alpha}\right) .
\end{aligned}
$$

By definition of $y_{\alpha}$ and $\mu_{\alpha}$, we have that $\mu_{\alpha} R_{\alpha}^{\prime}\left(y_{\alpha}\right)^{-1} \rightarrow 0$ when $\alpha \rightarrow+\infty$, and hence the right-hand-side of the above equation is positive. In case $i \notin I_{0}$, we get 
that

$$
\begin{gathered}
\lim _{\alpha \rightarrow+\infty} d_{g}\left(\exp _{\bar{y}_{\alpha}}\left(\mu_{\alpha} x\right), G_{i}^{\prime} x_{\alpha}^{i}\right)=d_{g}\left(G^{\prime} y_{0}, G_{i}^{\prime} x_{\infty}^{i}\right) \\
=d_{g}\left(G y_{0}, G x_{\infty}^{i}\right)>0 \text { in } C_{l o c}^{0}\left(\mathbb{R}^{n-k}\right) .
\end{gathered}
$$

Since $R_{\alpha}^{\prime}\left(y_{\alpha}\right) \rightarrow 0$ when $\alpha \rightarrow+\infty$, we then get that

$$
R_{\alpha}^{\prime}\left(\exp _{\bar{y}_{\alpha}}\left(\mu_{\alpha} x\right)\right) \geq \frac{1}{2}\left(1-\frac{\rho \mu_{\alpha}}{R_{\alpha}^{\prime}\left(y_{\alpha}\right)}\right) R_{\alpha}^{\prime}\left(y_{\alpha}\right)>0
$$

for all $x \in B_{0}(\rho)$ and all $\alpha>0$. We can then write for $x \in B_{0}(\rho)$ that

$$
\begin{aligned}
w_{\alpha}(x) & =\frac{\mu_{\alpha}^{\frac{n-k-p}{p}} v_{\alpha}\left(z_{\alpha}\right)}{R_{\alpha}^{\prime}\left(\exp _{\bar{y}_{\alpha}}\left(\mu_{\alpha} x\right)\right)^{\frac{n-k-p}{p}}} \\
& \leq 2^{(n-k-p) / p}\left(1-\frac{\rho \mu_{\alpha}}{R_{\alpha}^{\prime}\left(y_{\alpha}\right)}\right)^{-\frac{n-k-p}{p}} \frac{u_{\alpha}\left(y_{\alpha}\right)^{-1} v_{\alpha}\left(y_{\alpha}\right)}{R_{\alpha}^{\prime}\left(y_{\alpha}\right)^{\frac{n-k-p}{p}}} \\
& \leq 2^{(n-k-p) / p}\left(1-\frac{\rho \mu_{\alpha}}{R_{\alpha}^{\prime}\left(y_{\alpha}\right)}\right)^{-\frac{n-k-p}{p}}
\end{aligned}
$$

uniformly for $x \in B_{0}(\rho) \subset \mathbb{R}^{n-k}$ when $\alpha \rightarrow+\infty$. Thus the sequence $\left(w_{\alpha}\right)$ is uniformly bounded on every compact subset of $\mathbb{R}^{n-k}$. Let $\bar{g}_{\alpha}$ be the Riemannian metric on $\mathbb{R}^{n-k}$ defined by

$$
\bar{g}_{\alpha}(x)=\exp _{\bar{y}_{\alpha}}^{*} \bar{g}\left(\mu_{\alpha} x\right)
$$

Equation $\left(E_{\alpha}\right)$ becomes

$$
-\operatorname{div}_{\bar{g}_{\alpha}}\left(\tilde{v}_{\alpha}\left|\nabla w_{\alpha}\right|_{\bar{g}_{\alpha}}^{p-2} \nabla w_{\alpha}\right)+\mu_{\alpha}^{p} \tilde{h}_{\alpha} \tilde{v}_{\alpha} w_{\alpha}^{p-1}=\tilde{f}_{\alpha} \tilde{v}_{\alpha} w_{\alpha}^{p^{*}-1}
$$

where $\tilde{h}_{\alpha}(x)=\bar{h}_{\alpha}\left(\exp _{\bar{y}_{\alpha}}\left(\mu_{\alpha} x\right)\right), \tilde{f}_{\alpha}(x)=\bar{f}\left(\exp _{\bar{y}_{\alpha}}\left(\mu_{\alpha} x\right)\right), \tilde{v}_{\alpha}(x)=\bar{v}\left(\exp _{\bar{y}_{\alpha}}\left(\mu_{\alpha} x\right)\right)$. Since $\mu_{\alpha} \rightarrow 0$ when $\alpha \rightarrow+\infty$, the metric $\bar{g}_{\alpha}$ converges to the Euclidean metric $\xi$ in $C_{l o c}^{2}\left(\mathbb{R}^{n-k}\right)$ when $\alpha \rightarrow+\infty$. It then follows from Tolksdorff [13] that, up to extraction, there exists $w \in C^{1, \theta}\left(\mathbb{R}^{n-k}\right)$ such that

$$
\lim _{\alpha \rightarrow+\infty} w_{\alpha}=w \text { in } C_{l o c}^{1, \theta}\left(\mathbb{R}^{n-k}\right) .
$$

Since $w_{\alpha}(0)=1$, we get that $w(0)=1$ and then $w \neq \equiv 0$. We let $R>0$. Since

$$
\int_{B_{0}(R)} w_{\alpha}^{p^{*}} d v_{\bar{g}_{\alpha}}=\int_{B_{\bar{y}_{\alpha}}\left(R \mu_{\alpha}\right)} \bar{u}_{\alpha}^{p^{*}} d v_{\bar{g}}=\int_{B_{G^{\prime} y_{\alpha}}\left(R \mu_{\alpha}\right)} \operatorname{Vol}\left(G^{\prime} x\right)^{-1} u_{\alpha}^{p^{*}}(x) d v_{g}(x),
$$

we get that

$$
\lim _{\alpha \rightarrow+\infty} \int_{B_{G^{\prime} y_{\alpha}}\left(R \mu_{\alpha}\right)} u_{\alpha}^{p^{*}} d v_{g}=\operatorname{Vol}\left(G y_{0}\right) \int_{B_{0}(R)} w^{p^{*}} d v_{\xi}>0
$$


With the $H_{1}^{p}$ decomposition of Theorem 0.1, we then get that

$$
\begin{aligned}
1 & \leq C \int_{B_{G^{\prime} y_{\alpha}}\left(R \mu_{\alpha}\right)}\left(u^{0}+\sum_{i=1}^{l} B_{\alpha}^{i}+S_{\alpha}\right)^{p^{*}} d v_{g} \\
& \leq C \sum_{i=1}^{l} \int_{B_{G^{\prime} y_{\alpha}}\left(R \mu_{\alpha}\right)}\left(B_{\alpha}^{i}\right)^{p^{*}} d v_{g}+o(1) \\
& \leq C \sum_{i \in I_{0}} \int_{B_{G^{\prime} y_{\alpha}}\left(R \mu_{\alpha}\right)}\left(B_{\alpha}^{i}\right)^{p^{*}} d v_{g}+o(1) \\
& \leq C \sum_{i \in I_{0}} \int_{B_{\bar{y}_{\alpha}}\left(R \mu_{\alpha}\right)}\left(\bar{B}_{\alpha}^{i}\right)^{p^{*}} d v_{\bar{g}}+o(1)
\end{aligned}
$$

where, here again, we have taken the quotient wrt the group $G^{\prime}$ : this is licit since we work at the points $x_{\alpha}^{i}$ such that $x_{\infty}^{i}=y_{0}$. We can then prove exactly as in Saintier [12] that the right-hand side of this inequality goes to 0 as $\alpha \rightarrow+\infty$. A contradiction, and then (31) holds.

We claim that (30) holds. Indeed, the proof goes by contradiction and we consider a sequence of points $\left(y_{\alpha}\right)$ such that $\lim _{\alpha \rightarrow+\infty} R_{\alpha}(x)^{\frac{n-k-p}{p}} u_{\alpha}\left(y_{\alpha}\right)=+\infty$. With arguments similar to the ones above, we get that $\lim _{\alpha \rightarrow+\infty} y_{\alpha}=y_{0} \in M$ is such that $G y_{0}$ is an orbit of concentration of the $u_{\alpha}$ 's. Hypothesis $(\mathrm{H})$ yields a group $G^{\prime}$ that satisfies (H1) and (H2). With (H2), we get that $d_{g}\left(G y_{\alpha}, G x_{\alpha}^{i}\right) \leq$ $d_{g}\left(G^{\prime} y_{\alpha}, G^{\prime} x_{\alpha}^{i}\right)$ for the $i$ 's such that $\lim _{\alpha \rightarrow+\infty} x_{\alpha}^{i} \in G y_{0}$. Studying separately the remaining $i$ 's, we get that $R_{\alpha}\left(y_{\alpha}\right) \leq c R_{\alpha}^{\prime}\left(y_{\alpha}\right)$ and we apply (31) to get a contradiction with our initial assumption. This proves that (30) holds.

The proof of (11) goes the same way: if (11) is not satisfies, then we construct a sequence $\left(y_{\alpha}\right)$ which traducts it. We blow-up $u_{\alpha}$ at $y_{\alpha}$ and we get a contradiction as above.

\section{Acknowledgements}

The author would like to express his gratitude to Prof. Frédéric Robert for his careful reading and helpful comments.

\section{References}

[1] G. E. BREDON, Introduction to compact transformation groups, Academic Press, Pure and applied mathematics, 46, 1972.

[2] Z. FAGET, Best constant in Sobolev inequalities on Riemannian manifolds in the presence of symmetries, Potential Analysis, 17(2) (2002), 105-124.

[3] Z. FAGET, Optimal constants in critical Sobolev inequalities on Riemannian manifolds in the presence of symmetries, Ann. Global Anal. Geom., 24 (2003), $161-200$. 
[4] E. HEBEY, Nonlinear analysis on manifolds: Sobolev spaces and inequalities, Courant Lecture Notes in Mathematics, 51999.

[5] E. HEBEY and F. ROBERT, Coercivity and Struwe's compactness for Paneitz type operators with constant coefficients, Calc. Var. Partial Differential Equations, 13(4) (2001), 491-517.

[6] E. HEBEY and M. VAUGON, Sobolev spaces in the presence of symmetries, J. Math. Pures Appl., 76(10) (1997), 859-881.

[7] P. L. LIONS, The concentration-compactness principle in the calculus of variations, the limit case, parts 1 and 2, Rev.Mat.Iberoamericana, 1 (1985), 145-201, 45-121.

[8] F. ROBERT, Positive solutions for a fourth order equation invariant under isometries, Proc. Amer. Math. Soc., 131(5) (2003), 1423-1431.

[9] N. SAINTIER, Asymptotic estimates and blow-up theory for critical equations involving the $p$-Laplacian, Calc.Var. Partial Differential Equations, 25(3) (2006), 299-311.

[10] M. STRUWE, A global compactness result for elliptic boundary value problem involving limiting nonlinearities, Math. Z., 187(4) (1984), 511-517.

[11] M. STRUWE, Variational methods. Applications to nonlinear partial differential equations and Hamiltonian systems, Springer-Verlag, Berlin, 2000.

[12] P. TOLKSDORF, Regularity for a more general class of quasilinear elliptic equations, J. Differential Equations, 51(1) (1984), 126-150.

[13] J. L. VAZQUEZ, A strong maximum principle for some quasilinear elliptic equations, Appl. Math. Optim., 12(3) (1984), 191-202.

Received 5 February 2007; accepted 25 June 2007;

published Online First 10 January 2008

To access this journal online:

http://www.birkhauser.ch 